\title{
Cefmetazole for bacteremia caused by ESBL-producing enterobacteriaceae comparing with carbapenems
}

\author{
Takahiko Fukuchi ${ }^{1,2^{*}} \mathbb{D}$, Kentaro Iwata ${ }^{1,2}$, Saori Kobayashi ${ }^{3}$, Tatsuya Nakamura ${ }^{3}$ and Goh Ohji 1,2,3
}

\begin{abstract}
Background: ESBL (Extended spectrum beta-lactamase) producing enterobacteriaceae are challenging organisms with little treatment options. Carbapenems are frequently used, but the emergence of carbapenem resistant enterobacteriaceae is a concerning issue, which may hinder the use of carbapenems. Although cephamycins such as cefoxitin, cefmetazole or cefotetan are effective against ESBL-producers in vitro, there are few clinical data demonstrating effects against bacteremia caused by these organisms.
\end{abstract}

Methods: We performed a retrospective observational study on cases of bacteremia caused by ESBL-producers to investigate the efficacy of cefmetazole compared with carbapenems. We also evaluated whether the trend of antibiotic choice changed over years.

Results: Sixty-nine patients (male 34, age 69.2 \pm 14.4 ), including two relapse cases, were reviewed for this analysis. The most common causative organisms were Escherichia coli (64, 93 \%), followed by Klebsiella pneumoniae and K. oxytoca (2 each, $4 \%)$. The group that received carbapenem therapy $(43,62 \%)$ had increased severity in the Pittsburgh Bacteremic score than the group that received cefmetazole therapy, (1.5 \pm 1.5 vs $2.5 \pm 2.1, p=0.048)$, while analysis of other factors didn't reveal any statistical differences. Five patients in the carbapenem group and one patient in the cefmetazole group died during the observation period $(p=0.24)$. CTX-M-9 were predominant in this series (59\%). Infectious disease physicians initially recommended carbapenems at the beginning of the current research period, which gradually changed over time favoring the use of cefmetazole instead $(p=0.002)$.

Conclusion: Cefmetazole may be safely given to patients with bacteremia caused by ESBL-producers as a definitive therapy, if one can select out relatively stable patients.

Keywords: ESBL, Extended spectrum beta-lactamase, Bacteremia, Cephamycin, Cefmetazole

Abbreviations: CMZ, Cefmetazole; CPs, Carbapenems; CRE, Carbapenems resistant enterobacteriaceae; ESBL, Extended spectrum beta-lactamase

\section{Background}

Spreading antibiotic resistance is a global concern. In terms of enterobacteriaceae, extended spectrum beta lactamase (ESBL) producing enterobacteriaceae and carbapenem resistant enterobacteriaceae (CRE) are the most concerning pathogens [1]. Infections caused by

\footnotetext{
* Correspondence: chicco@f.email.ne.jp

${ }^{1}$ Division of Infectious Diseases Therapeutics, Kobe University Graduate School of Medicine, 7-5-2 Kusunokicho, Chuoku, Kobe, Hyogo 650-0017, Japan

${ }^{2}$ Department of Microbiology and Infectious Disease, Kobe University

Graduate School of Medicine, 7-5-2 Kusunokicho, Chuoku, Kobe, Hyogo

650-0017, Japan

Full list of author information is available at the end of the article
}

ESBL producing enterobacteriaceae (ESBL-E) are associated with higher mortality [2] and medical cost [3-5].

There is a strong relationship between antibiotic use and increase in prevalence of resistant pathogens. The drugs most often used against ESBL-producers are carbapenems [6]. Research has elucidated that recent exposure (3 months prior) to antibiotics was a parameter consistently associated with detection of CRE [7, 8]. Overuse of carbapenems and subsequent selective pressure can contribute to the spread of CRE. CREs are rarely identified in Japan as of this writing, but overreliance on carbapenems may induce CREs. Thus, 
CRE became an emerging pathogen, especially in Europe and North America.

Cephamycins, such as cefmetazole and flomoxef have good activity against ESBL-producers in vitro. However, clinical data regarding the potential value of cephamycins for the treatment of ESBL-associated infections are scarce [9]. Some are also reluctant to use cephamycins as first-line therapy for ESBL-producing organisms [10].

We therefore conducted a study to evaluate the efficacy of cefmetazole against bacteremia caused by ESBLE. In addition, the trend of the prescription of antibiotics against ESBL-E bacteremia were evaluated over years to see the effectiveness of Infectious Disease consultants in avoiding the overuse of carbapenems.

\section{Methods}

We conducted a retrospective observational study at a 930-bed university hospital in Kobe, Japan. All patients admitted from 1st January 2008 through until 31 December 2013 who had ESBL-E isolated from blood cultures were identified. The clinical records of all 125 who had positive blood cultures of ESBL-E, and those who were treated with carbapenems or cefmetazole as definitive therapy were reviewed. ESBL-E were defined as positive strains of the double disc synergy test [11].

Duplicated cultures, pediatric patients, those judged as contamination by the infectious disease team, and palliative care patients were excluded.

The following data was collected from the clinical records; age, gender, community acquired or nosocomial infection, origin of bacteremia, causative pathogens, comorbidities (using Charlson comorbidity scoring index; myocardial infarction, congestive heart failure, peripheral vascular disease, dementia, chronic pulmonary disease, connective tissue disease, peptic ulcer disease, liver disease, diabetes mellitus, hemiplesia, renal disease, tumor or lymphoma with or without metastasis, leukemia, and AIDS), vital signs (using Pittsburgh bacteremic Score and SOFA score), admission into ICU, bacterial profile (single pathogen or mixed infections, resistance against cefmetazole, resistance against fluoroquinolones), treatment profile (empirical treatment, definitive treatment, consultation with infectious disease physicians, the efficacy of empirical antibiotics).

Primary outcomes were defined as a death within 30 days after the documentation of bacteremia and a relapse of bacteremia caused by the same pathogen.

In addition, we evaluated the relationship between treatment options and consultation with infectious disease physicians over years. Furthermore, we estimated the resistant genes of ESBL-E. Genetic detection and genotyping of TEM, SHV, and CTX-M were performed by using PCR with bacterial DNA, which was extracted from the isolates by boiling the bacterial suspensions. A solution with an extracted DNA concentration of $0.1 \mathrm{ng} /$ $\mathrm{mL}$ was used as the template for PCR analysis. In the case of genotyping of CTX-M genes, 4 primer sets that amplify group-specific CTX-M genes were used, as described previously: the CTX-M1 group includes CTXM-1, CTX-M-3, CTX-M-10 to CTX-M-12, CTX-M-15, CTX-M-22, CTX-M-23, and CTX-M-28 to CTX-M-30; the CTX-M2 group, CTX-M-2, CTX-M-4 to CTX-M-7, CTX-M-20, and Toho-1; the CTX-M8 group, CTX-M-8; and the CTX-M9 group, CTX-M-9, CTX-M-13, CTXM-14, CTX-M-16 to CTX-M-19, CTX-M-21, CTX-M27, and Toho-2. The PCR products were analyzed using $2 \%$ agarose gel electrophoresis and visualized by staining with ethidium bromide.

The statistical analysis was performed using STATA 13 (STATA corp. LP, College Station, Texas, USA). The Mann-Whitney test was used to compare the two groups, also an extension to the Wilcoxon rank-sum test, the 'nptrend' STATA command, was used to assess trends in proportions across ordered groups. All test were two-tailed and $p$ values $\leq 0.05$ were used for statistical significance testing.

\section{Result}

Sixty-nine patients with ESBL-E bacteremia were reviewed for the analysis (Fig. 1). Twenty-six (38 \%) patients were given cefmetazole (CMZ) as definitive therapy, while 43 patients (62\%) were given carbapenems (CPs), of which meropenem was used most (39), followed by doripenem (3), and biapenem (1). The average age of the patients was $67.5 \pm 14.6$ and $71.2 \pm$ 13.0 in CMZ group and CPs group, respectively (Table $1, P=0.18)$. SOFA score tended to be higher in CPs group, although it did not reach statistical significance $(P=0.055)$. The Pittsburgh Bacteremic Score in the CPs group was significantly higher than CMZ group $(P=0.048)$. The most frequent origin of infection was the urinary tract in both groups (CMZ group: 18, $69 \%$, CPs group: 17, $40 \%$ ), followed by hepatobiliary infections (CMZ group: 5, 20 \%, CPs group: 8, $19 \%$ ). The most frequently isolated pathogens were Escherichia coli in both groups (CMZ group: 25, $96 \%$, CPs group: 39, $91 \%$ ). The CMZ group had one case caused by Klebsiella pneumoniae, and the CPs group had two $K$. oxytoca and one $K$. penumoniae. The most used empiric therapies in the CPs group were carbapenems (27; meropenem 21, doripenem 3, biapenem 2, imipenem/ cilastatin 1), followed by cefazolin (5), ceftriaxone (4), sulbactam/cefoperazone (3) (Table 2). The most used empiric therapies in the CMZ group were meropenem (6) followed by cefepime (4), tazobactam/piperacillin (4), sulbactam/ampicillin (3), ceftriaxone (3), cefmetazole (2). Antibiotic coverage rates are shown in Table 1. Genetic analysis revealed CTX-M-9 was predominant 


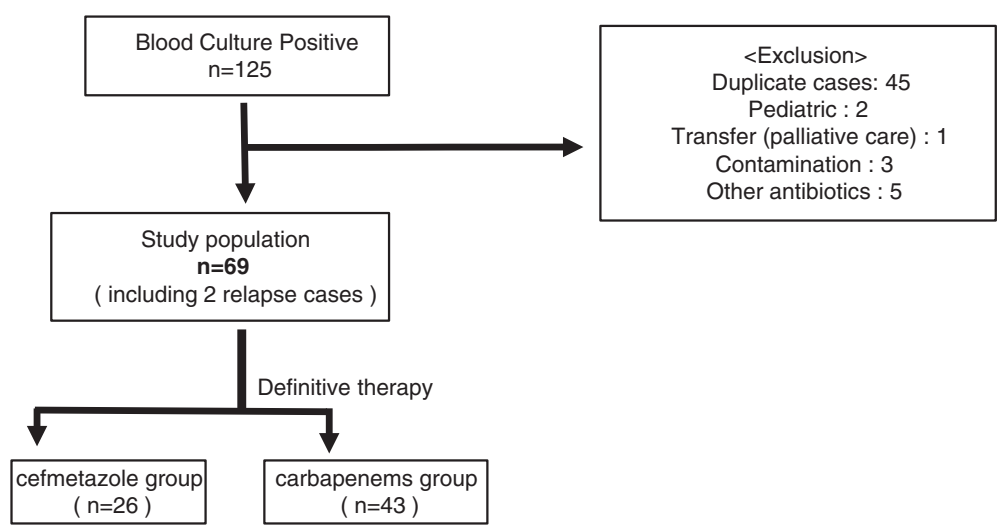

Fig. 1 Enrollment of the patients

in isolated strains in this series $(39,59 \%)$, followed by CTX-M-1 (21, $32 \%)$, M2, TEM/SHV, KOXY (2 each, $3 \%$ ).

Six patients died during the study period (CMZ group: 1, CPs group: 5), while 2 patients in the CPs group relapsed (became bacteraemic) after treatment with meropenem. As a result, $96 \%$ of patients in the CMZ group and $84 \%$ of patients in the CPs group survived without relapse $(p=0.24)$.

The CPs group and the CMZ group had resistant genes such as CTX-M9 $(20,16)$, CTX-M1 $(13,7)$, CTX-

Table 1 The Baseline Characteristics of the Patients

\begin{tabular}{|c|c|c|c|c|}
\hline & & $\begin{array}{l}\text { Cefmetazole } \\
\text { group }\end{array}$ & $\begin{array}{l}\text { Carbapenems } \\
\text { group }\end{array}$ & $p$ value \\
\hline & number & 26 & 43 & - \\
\hline & male & 12 & 22 & - \\
\hline & age & 67.5 & 71.2 & - \\
\hline Source & UTI & 18 (69 \%) & 17 (40 \%) & - \\
\hline Pathogen & E.coli & 25 (96 \%) & 39 (91%) & - \\
\hline \multirow[t]{3}{*}{ Outcome } & $\begin{array}{l}\text { survive without } \\
\text { relapse }\end{array}$ & 25 & 36 & 0.243 \\
\hline & death & 1 & 5 & \\
\hline & relapse & 0 & 2 & \\
\hline \multirow{5}{*}{$\begin{array}{l}\text { Patient } \\
\text { profile }\end{array}$} & SOFA & $2.8+-2.8$ & $5.0+-4.5$ & 0.055 \\
\hline & Charlson & $7.1+-3.4$ & $6.8+-2.8$ & 0.85 \\
\hline & Pitt. Bact. Score & $1.5+-1.5$ & $2.5+-2.1$ & 0.048 \\
\hline & admission to ICU & $5(24 \%)$ & 17 (39 \%) & 0.111 \\
\hline & $\begin{array}{l}\text { community } \\
\text { acquired }\end{array}$ & 10 (38 \%) & $11(26 \%)$ & 0.29 \\
\hline \multirow{3}{*}{$\begin{array}{l}\text { Bacterial } \\
\text { profile }\end{array}$} & mixed infection & $2(8 \%)$ & $7(16 \%)$ & 0.466 \\
\hline & cefmetazole:R & $0(0 \%)$ & $1(2 \%)$ & 1 \\
\hline & fluoroquinolone:R & 19 (73 \%) & 34 (79 \%) & 1 \\
\hline \multirow{2}{*}{$\begin{array}{l}\text { Treatment } \\
\text { profile }\end{array}$} & ID consult & 19 (73 \%) & 21 (49 \%) & 0.077 \\
\hline & empiric cover & 13 (50 \%) & 27 (63 \%) & 0.325 \\
\hline
\end{tabular}

M2 (1, 1), KOXY (2, 0), TEM/SHV (2, 0), respectively. Five patients in the CPs group died, and their ESBLs were CTX-M9 (1), CTX-M1 (3), KOXY (1).

Resistance against cefmetazole was rare (only 1 case in CPs group, or $1 \%$ ).

The consultation to infectious disease physicians tended to be higher in the CMZ group, ( $75 \%$ vs $49 \%, p=0.077$ ).

The selection of antibiotics, ID consultation, and outcomes of each-year are shown in Fig. 2. The CMZ group and the CPs group had a significant difference in trend over years with the use of CMZ becoming much more popular recently $(p=0.02)$.

\section{Discussion}

This observational study demonstrated that bacteremia caused by ESBL-E were effectively treated with cefmetazole as definitive therapy, and this suggests cefmetazole can be a candidate as an alternative for carbapenems. The patients in both groups had low mortality. Of note, relapse of bacteremia caused by ESBL-producer occurred only in the carbapenems group, although these relapsed patients were originally treated with sufficient quantity and duration of carbapenems.

Over years both cases with and without Infectious Diseases physician consults started to use CMZ as the definitive therapy probably due to confidence in the use of CMZ against ESBL-E (Fig. 2).

The rationale of antimicrobial therapy against sepsis or bacteremia is as follows. As empirical therapy, broadspectrum antibiotics must be selected with one or more agents active against likely bacterial pathogens after obtaining appropriate cultures. It should be reevaluated according to the results of cultures to optimize efficacy, to prevent resistance, to avoid toxicity, and to minimize costs [12]. When treating ESBL-producers, carbapenems have unnecessary coverage against non-fermenters such as Pseudomonas and Acinetobacter, which would make carbapenems unfavorable antibiotics. 
Table 2 Empiric therapies and proportion of unfavorable outcomes on both groups of cefmetazole (CMZ) and carbapenems (CPs)

\begin{tabular}{|c|c|c|c|c|c|c|c|}
\hline \multicolumn{4}{|l|}{ CMZ group } & \multicolumn{4}{|l|}{ CPs group } \\
\hline & Empiric therapy & Number & $\begin{array}{l}\text { Unfavorable } \\
\text { outcomes }\end{array}$ & & $\begin{array}{l}\text { Empiric } \\
\text { therapy }\end{array}$ & Number & $\begin{array}{l}\text { Unfavorable } \\
\text { outcomes }\end{array}$ \\
\hline \multirow{3}{*}{$\begin{array}{l}\text { Empiric therapy effective } \\
\text { against the causative } \\
\text { organisms }\end{array}$} & CPs & 7 & 1:died & \multirow{3}{*}{$\begin{array}{l}\text { Empiric therapy effective } \\
\text { against the causative } \\
\text { organisms }\end{array}$} & \multirow[t]{3}{*}{ CPs } & \multirow[t]{3}{*}{27} & \multirow{3}{*}{$\begin{array}{l}\text { 3:died, } \\
\text { 2:relapsed }\end{array}$} \\
\hline & tazobactam/piperacillin & 4 & none & & & & \\
\hline & CMZ & 2 & & & & & \\
\hline \multirow{4}{*}{$\begin{array}{l}\text { Empiric therapy ineffective } \\
\text { against the causative } \\
\text { organisms }\end{array}$} & cefepime & 4 & \multirow[t]{4}{*}{ none } & \multirow{4}{*}{$\begin{array}{l}\text { Empiric therapy ineffective } \\
\text { against the causative } \\
\text { organisms }\end{array}$} & cefazolin & 5 & 1:died \\
\hline & ceftriaxone & 3 & & & ceftriaxone & 4 & 1:died \\
\hline & sulbactam/ampicillin & 3 & & & $\begin{array}{l}\text { sulbactam/ } \\
\text { cefoperazone }\end{array}$ & 3 & \multirow[t]{2}{*}{ none } \\
\hline & others & 3 & & & Others & 2 & \\
\hline
\end{tabular}

Cefmetazole has a narrower spectrum compared to carbapenems and does not cover Pseudomonas or Acinetobacter. These characteristics would provide another advantage of cefmetazole over carbapenems. Cefmetazole can play an important role in antimicrobial stewardship programs.

Studies have shown the efficacy of cefmetazole against ESBL producing enterobacteriaceae. Matsumura et al. found that treatment with cefmetazole or flomoxef against bacteremia caused by ESBL-producing E. coli were similarly effective compared with treatment with carbapenems in a retrospective multicenter study using a propensity score-adjusted analysis [13]. This study demonstrated the efficacy of two cephamycins against ESBL-producing E.coli in both empirical and definitive therapy cohorts. However, use of cefmetazole as an empirical therapeutic agent is not realistic, because one cannot predict whether the causative pathogens were really ESBL-E prior to the culture result. Therefore, we rather tried to focus on definitive therapy alone, where cefmetazole could be utilized better.

Doi A et al. showed the efficacy of cefmetazole against pyelonephritis caused by ESBL-producing enterobacteriaceae [14]. Bacteremic patients were not included in the CMZ group in this study (0/7), as opposed to carbapenems (8/12). Our study focused on bacteremic patients, which makes our results applicable in a wider setting.

Considering other candidates, there are various studies claiming the efficacy of beta-lactam/beta-lactamase inhibitor (BLBLI) against ESBL-producers, but with some controversies. Piperacillin/tazobactam is a promising antibiotic, although piperacillin has unnecessary coverage against Pseudomonas. In addition, the role of piperacillin/tazobactam for patients with ESBL-E remains unclear. A meta-analysis described BLBLI as being non inferior compared to carbapenems [2]. However, some research in the US and Taiwan and a cohort study in the US showed higher mortality when piperacillin/tazobactam is used when compared with carbapenems $[6,15,16]$.
On the other hand, ertapenem has good activity against ESBL producers, and has the advantage of not covering Pseudomonas, a different characteristic from other carbapenems [17]. Ertapenem is still much broader than CMZ, making CMZ more advantageous over ertapenem. Ertapenem use led to the selection of multi-drug resistant bacteria at a Singapore hospital [18].

By the same token, cefepime could be effective against the strains in North America. In East Asia, however, it has less activity against ESBL-E (78.6 \%) [19]. Similarly, aminoglycosides keep their activity against ESBL-E. Despite this, many physicians are reluctant to use aminoglycosides for fear of adverse effects such as nephrotoxicity [20].

Regarding the diversity of ESBL-E, there are at least 300 strains in the world. Taiwanese research revealed high resistance of ESBL-E against CMZ (40 \%), but most were CTX-M-14 or CTX-M-15 [21]. In our study, CTXM-9 was the most common strain (51\%).

There are several limitations in this study. First, this is only a retrospective observational study at one institution. Resistance patterns differ among hospitals, regions, or countries. Our results might not be applied to other settings, particularly where the genotype of ESBL producers are different. However, settings with similar resistant patterns to our hospital may use CMZ like our practice. Secondly, our patients had relatively less severe infection than those in the previous studies. Third, selection bias could not be completely removed in both groups.

Even though we are aware of these limitations, the fact that most patients with bacteremia caused by ESBLproducers were successfully treated with cefmetazole was quite promising. For clinically stable patients, who are already improving on empirical antimicrobial treatment, or without clinical deterioration can be a good candidate for de-escalation to cefmetazole with close clinical monitoring. Prospective studies in various settings may confirm our findings in the future. 


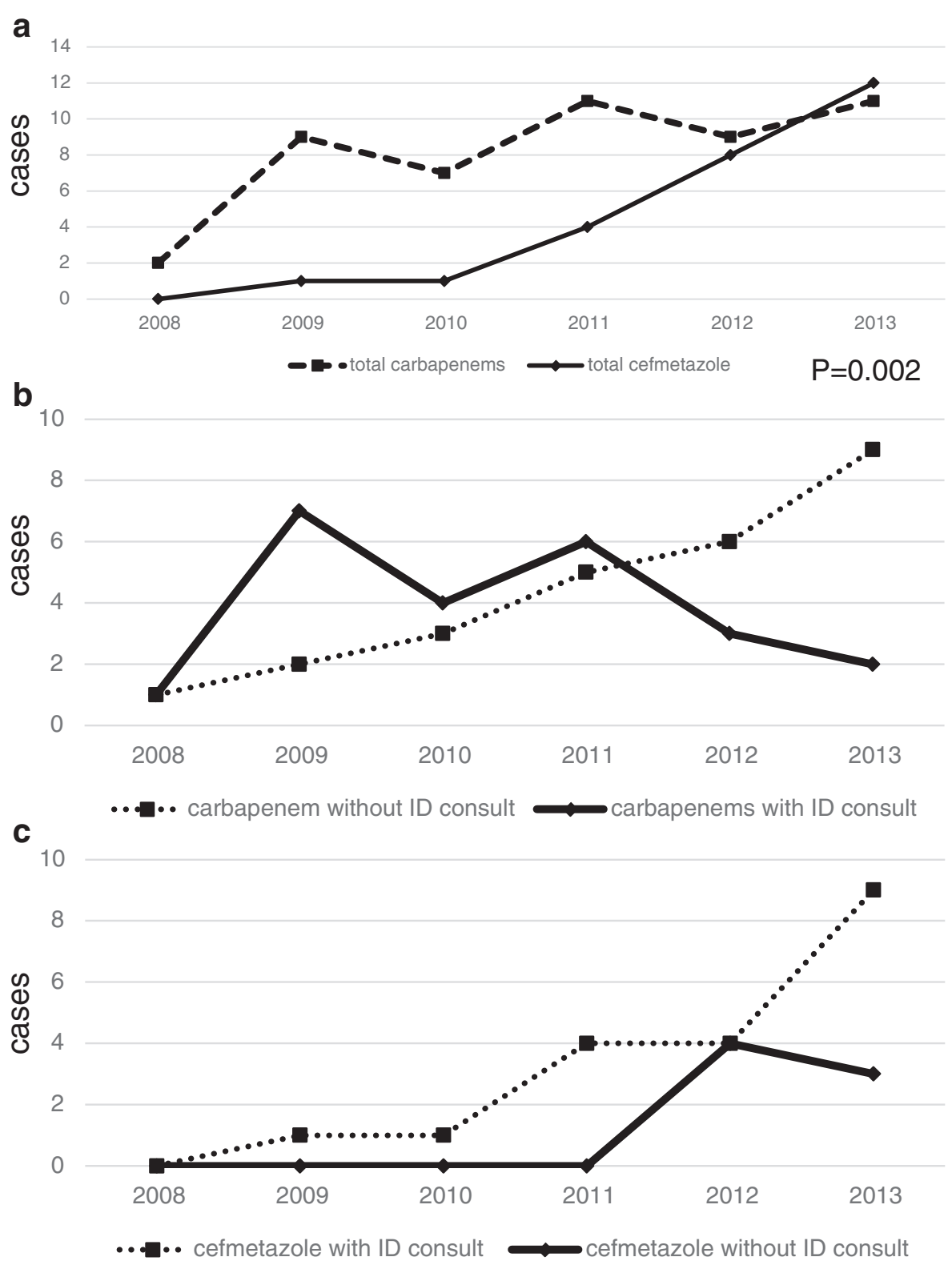

Fig. $\mathbf{2}$ a use of study antimicrobials by year. b The number of the patients using carbapenems with or without ID consultation. c The number of the patients using cefmetazole with or without ID consultation

\section{Conclusion}

Our study suggests cefmetazole may be effective against bacteremia due to ESBL-producing Enterobacteriaceae as a definitive therapy, therefore allowing the sparing of carbapenems against the organisms. Further research, such as prospective interventional studies are needed to confirm our findings.

\section{Acknowledgements}

This study was presented in 2014 IMED Vienna, Austria in 2014.

The authors would like to acknowledge all of the laboratory staff at

Department of Clinical Laboratory, Kobe University Hospital, Kobe, JAPAN.

\section{Funding}

There is no source of funding.

Availability of data and materials

All data containing relevant information to support the study findings are provided in the manuscript.

\section{Authors' contribution}

TF performed data collection, data analysis, interpretation, and drafted the manuscript. IK designed the study, and contributed interpretation, coordination, and reviewing of the manuscript. KS and NT performed microbiological experiments. OG helped to the draft manuscript. All authors read and approved the final version. 


\section{Competing interests}

The authors declare that they have no competing interests.

\section{Consent for publication}

Not applicable.

\section{Ethics approval and consent to participate}

The ethics committee at Kobe University Graduate School of Medicine approved this study. The committee approved this study without the need for written informed consent because the data lacked patient identifiers.

\section{Author details}

'Division of Infectious Diseases Therapeutics, Kobe University Graduate Schoo of Medicine, 7-5-2 Kusunokicho, Chuoku, Kobe, Hyogo 650-0017, Japan. 2Department of Microbiology and Infectious Disease, Kobe University Graduate School of Medicine, 7-5-2 Kusunokicho, Chuoku, Kobe, Hyogo 650-0017, Japan. ${ }^{3}$ Department of Clinical Laboratory, Kobe University Hospital, 7-5-2 Kusunokicho, Chuoku, Kobe, Hyogo 650-0017, Japan.

Received: 13 November 2015 Accepted: 10 August 2016 Published online: 18 August 2016

\section{References}

1. Signs V. Carbapenem-Reistant Enterobacteriaceae. MMWR. 2013;62:165-70.

2. Vardakas KZ, Tansarli GS, Rafailidis Pl, Falagas ME. Carbapenems versus alternative antibiotics for the treatment of bacteraemia due to Enterobacteriaceae producing extended-spectrum beta-lactamases: a systematic review and meta-analysis. J Antimicrob Chemother. 2012;67(12): 2793-803.

3. Force TBART. The Cost of Antibiotic Resistance: Effect of Resistance Among Staphylococcus aureus, Klebsiella pneumoniae, Acinetobacter baumannii, and Pseudomonas aeruginosa on Length of Hospital Stay. Infection Control and Hospital Epideimology. 2002;23:106-8

4. Schwaber MJ, Carmeli Y. Mortality and delay in effective therapy associated with extended-spectrum beta-lactamase production in Enterobacteriaceae bacteraemia: a systematic review and meta-analysis. J Antimicrob Chemother. 2007:60(5):913-20.

5. Leistner R, Gurntke S, Sakellariou C, Denkel LA, Bloch A, Gastmeier P, et al. Bloodstream infection due to extended-spectrum beta-lactamase (ESBL)positive K. pneumoniae and E. coli: an analysis of the disease burden in a large cohort. Infection. 2014;42(6):991-7.

6. Paterson D. Antibiotic Therapy for Klebsiella pneumoniae Bacteremia: Implications of Production of Extended-Spectrum B-Lactamase. Clin Infect Dis. 2003:39:31-7.

7. Marchaim D, Chopra T, Bhargava A, Bogan C, Dhar S, Hayakawa K, et al. Recent exposure to antimicrobials and carbapenem-resistant Enterobacteriaceae: the role of antimicrobial stewardship. Infect Control Hosp Epidemiol. 2012:33(8):817-30.

8. Tumbarello M, Spanu T, Di Bidino R, Marchetti M, Ruggeri M, Trecarichi EM, et al. Costs of bloodstream infections caused by Escherichia coli and influence of extended-spectrum-beta-lactamase production and inadequate initial antibiotic therapy. Antimicrob Agents Chemother. 2010;54(10):4085-91.

9. Falagas ME, Karageorgopoulos DE. Extended-spectrum beta-lactamaseproducing organisms. J Hosp Infect. 2009;73(4):345-54.

10. Paterson DL, Bonomo RA. Extended-spectrum beta-lactamases: a clinical update. Clin Microbiol Rev. 2005;18(4):657-86.

11. Jarlier V, Nicolas MH, Fournier G, Philippon A. Extended broad-spectrum $\beta$ lactamases conferring transferable resistance to newer $\beta$-lactam agents in Enterobacteriaceae: hospital prevalence and susceptibility patterns. Rev Infect Dis. 1988;10:867-78.

12. Kumar A. Optimizing antimicrobial therapy in sepsis and septic shock. Crit Care Clin. 2009;25:733-51.

13. Matsumura $Y$, Yamamoto $M$, Nagao M, Komori T, Fujita N, Hayashi A, et al. Multicenter Retrospective Study of Cefmetazole and Flomoxef for Treatment of Extended-Spectrum-beta-Lactamase-Producing Escherichia coli Bacteremia. Antimicrob Agents Chemother. 2015;59(9):5107-13.

14. Doi A, Shimada T, Harada S, Iwata K, Kamiya T. The efficacy of cefmetazole against pyelonephritis caused by extended-spectrum beta-lactamaseproducing Enterobacteriaceae. Int J Infect Dis. 2013;17(3):e159-63.

15. Tsai HY, Chen YH, Tang HJ, Huang CC, Liao CH, Chu FY, et al. Carbapenems and piperacillin/tazobactam for the treatment of bacteremia caused by extended-spectrum beta-lactamase-producing Proteus mirabilis. Diagn Microbiol Infect Dis. 2014:80(3):222-6.

16. Tamma PD, Han JH, Rock C, Harris AD, Lautenbach E, Hsu AJ, et al. Carbapenem therapy is associated with improved survival compared with piperacillin-tazobactam for patients with extended-spectrum beta-lactamase bacteremia. Clin Infect Dis. 2015;60(9):1319-25.

17. Fong JJ, Rosé L, Radigan EA. Clinical outcomes with ertapenem as a first-line treatment option of infections caused by extended-spectrum $\beta$-lactamase producing gram-negative bacteria. Ann Pharmacother. 2012;46(3):347-52.

18. Lim C. Evaluation of Ertapenem use with Impact Assessment on Extended Spectrum Beta-Lactamase (ESBL) Production and Gram-Negative resistance in Singapore General Hospital (SGH). BMC Infectious Disease. 2013;13:523.

19. Kang Cl, Cha MK, Kim SH, Ko KS, Wi YM, Chung DR, et al. Clinical and molecular epidemiology of community-onset bacteremia caused by extended-spectrum beta-lactamase-producing Escherichia coli over a 6-year period. J Korean Med Sci. 2013;28(7):998-1004.

20. Park SH, Choi SM, Chang YK, Lee DG, Cho SY, Lee HJ, et al. The efficacy of non-carbapenem antibiotics for the treatment of community-onset acute pyelonephritis due to extended-spectrum beta-lactamase-producing Escherichia coli. J Antimicrob Chemother. 2014;69(10):2848-56.

21. Chung HC, Lai CH, Lin JN, Huang CK, Liang SH, Chen WF, et al. Bacteremia caused by extended-spectrum-beta-lactamase-producing Escherichia coli sequence type ST131 and non-ST131 clones: comparison of demographic data, clinical features, and mortality. Antimicrob Agents Chemother. 2012; 56(2):618-22.

\section{Submit your next manuscript to BioMed Central and we will help you at every step:}

- We accept pre-submission inquiries

- Our selector tool helps you to find the most relevant journal

- We provide round the clock customer support

- Convenient online submission

- Thorough peer review

- Inclusion in PubMed and all major indexing services

- Maximum visibility for your research

Submit your manuscript at www.biomedcentral.com/submit
) Biomed Central 